\title{
Femtosecond laser-induced optical anisotropy in a two-dimensional lattice of magnetic dots
}

\author{
I. Razdolski, ${ }^{1}$ V. L. Krutyanskiy, ${ }^{2}$ T. V. Murzina, ${ }^{2}$ Th. Rasing, ${ }^{1}$ and A. V. Kimel ${ }^{1}$ \\ ${ }^{1}$ Radboud University Nijmegen, Institute for Molecules and Materials, Heyendaalseweg 135, 6525 AJ Nijmegen, The Netherlands \\ ${ }^{2}$ Department of Physics, Moscow State University, 119991 Moscow, Russia
}

(Received 20 November 2013; revised manuscript received 30 January 2014; published 21 February 2014)

\begin{abstract}
Using pump-probe optical polarimetry we demonstrate that femtosecond laser excitation of a 2D regular lattice of magnetic nanodots effectively changes the optical anisotropy of the array. Study of the dynamics of the femtosecond laser-induced anisotropy reveals four main mechanisms occurring in the electronic, spin, and lattice subsystems. Below 1 ps, a strong Kerr-like nonlinearity causes linear birefringence, with its axis directed along the electric field of the linearly polarized femtosecond laser pump pulse. In addition, a long-living linear birefringence is also induced due to slowly relaxing excitations. Also below $1 \mathrm{ps,} \mathrm{ultrafast} \mathrm{laser-induced}$ demagnetization of Co leads to a partial breakdown of the circular birefringence of the magnetic nanodots. On the timescale up to $300 \mathrm{ps,} \mathrm{optically} \mathrm{triggered} \mathrm{acoustic} \mathrm{modes} \mathrm{of} \mathrm{the} \mathrm{dots} \mathrm{drive} \mathrm{oscillations} \mathrm{of} \mathrm{the} \mathrm{linear} \mathrm{optical}$ birefringence. During this process, the oscillations damp while transferring their energy into acoustic modes of the substrate. On the nanosecond timescale, the signal is dominated by acoustic oscillations at the surface of the substrate.
\end{abstract}

DOI: 10.1103/PhysRevB.89.064306

\section{INTRODUCTION}

The optical properties of regular arrays of nanosized objects demonstrate a large variety of interesting effects, involving entangled underlying physical processes [1-5]. There are a number of papers in this field tackling the problems of magneto-optics [6-8], opto-acoustics [9,10], and plasmonics [11-13], often extending the experimental approaches into the nonlinear domain [14] or employing time-resolved techniques [15-19].

A conventional approach to studying arrays of nanoobjects deals with optical reflectivity $[20,21]$ or nonlinear-optical conversion [22-24], allowing us to find such interesting phenomena as coherent control of optical transmission [25], enhancement of the up-conversion luminescence intensity [26], and periodicity-induced pulse splitting [27]. On the other hand, much less attention is drawn towards polarization effects which are capable of revealing the dynamics of the optical anisotropy induced by a fs laser pulse.

In this paper, we rigorously study polarization effects in a rectangular array of Co nanodots by means of a pumpprobe technique. Our analysis of the transient dynamics reveals several mechanisms responsible for the polarization rotation of the probe beam. At subpicosecond delay times a Kerr-type nonlinearity, together with a rapid laser-induced demagnetization, plays a dominant role in the polarization rotation. Later on, the excess heat in the nanodots triggers their acoustic eigenmodes, which decay while the heat is transferred into the substrate. On an even longer timescale, phononic eigenmodes of the periodic array of the dots are excited, modulating the polarization rotation of the probe beam with frequencies in the $\mathrm{GHz}$ range.

The paper is organized as follows. After briefly mentioning experimental methods and setup (Sec. II), we discuss a typical dynamical curve of polarization rotation of the probe beam and break it into three main time regions. We examine each of this regions closely in Section III, where we also discuss the main features of the effects observed on their various relevant timescales and analyze our experimental findings using a
PACS number(s): 63.22.-m, 78.47.J-, 78.20.Ls

phenomenological description of the dynamical birefringence observed at longer time delays. The Appendix section concludes the paper, providing some mathematical steps necessary to back up our phenomenology.

\section{SAMPLES AND EXPERIMENTAL PROCEDURE}

Two-dimensional square lattices of 30-nm-thick cobalt dots were fabricated by electron beam lithography and lift-off technique on a glass plate. The experimental procedure is described in more detail elsewhere [28]. The period $a$ of the lattice was $1.4 \mu \mathrm{m}$ and the typical size of the dots $d$ was about $0.6 \mu \mathrm{m}$.

The time-resolved experiments were performed employing a pump-probe technique where the powerful pump pulses (wavelength $800 \mathrm{~nm}$, pulse duration $80 \mathrm{fs}$, fluence $2.6 \pm$ $0.3 \mathrm{~mJ} / \mathrm{cm}^{2}$ ) excited the sample, and about 100 times less intense probe pulses at $500 \mathrm{~nm}$ were used to study polarization effects in the sample at a certain time delay. The angles of incidence were $0^{\circ}$ for the pump and $15^{\circ}$ for the probe beam. The pump and probe beams were focused at the surface of the sample into overlapping spots of approximately $60 \mu \mathrm{m}$ and $30 \mu \mathrm{m}$ in diameter, respectively. An external magnetic field of about $2 \mathrm{kOe}$ was applied in the longitudinal Kerr geometry (see Fig. 1), enough to saturate the sample.

In order to study pump-induced optical anisotropy in the magnetic lattice, we have measured the polarization rotation of the probe beam transmitted or reflected from the sample. Consider an isotropic medium, the optical properties of which are described by the dielectric permittivity tensor $\hat{\varepsilon}$. For such a medium one can always choose the coordinate system such that the dielectric permittivity tensor $\hat{\varepsilon}$ acquires a diagonal form. For the $X O Y$ plane, which coincides with the surface of the sample (see Fig. 1), one can write (where $\varepsilon_{x x}=\varepsilon_{y y}$ ):

$$
\hat{\varepsilon}=\left(\begin{array}{cc}
\varepsilon_{x x} & 0 \\
0 & \varepsilon_{x x}
\end{array}\right) .
$$




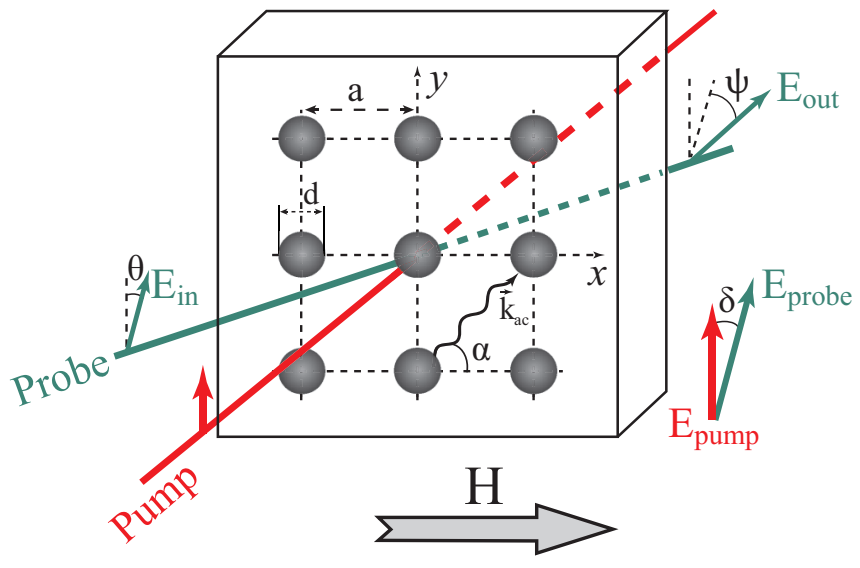

FIG. 1. (Color online) Schematic of the pump-probe experiment. The (XOY) plane corresponds to the surface of the sample, a $d c$ magnetic field is applied in the longitudinal Kerr geometry along the $(\mathrm{OX})$ axis; $\theta$ is the angle between the polarization plane of the incident probe beam and the (OY) axis, and $\psi$ is the angle between the polarization planes of the incident and transmitted (reflected) probe beams. Co nanodots of a diameter $d$ in the array are spatially separated by a lattice period $a$. An acoustic wave with the wave vector $\vec{k}_{a c}$ propagates at an angle $\alpha$ to the (OX) axis. On the right hand side the polarization difference angle $\delta$ between the pump and probe beams is shown.

Assume the pump excitation results in a photoinduced change of the optical properties leading to the anisotropy. This can happen in two ways, namely, the off-diagonal components $\varepsilon_{x y}=\varepsilon_{y x}$ can arise, or, alternatively, the degeneracy between $O X$ and $O Y$ directions can be lifted, so that $\varepsilon_{x x} \neq \varepsilon_{y y}$. If the probe beam at a small angle of incidence is polarized at an arbitrary angle $\theta$ with respect to the main axes (see Fig. 1), such an anisotropy will result in polarization rotation of the reflected or transmitted beam. The general equation (see Appendix) is bulky and can be obtained by means of analyzing the boundary conditions for the electric field and magnetic induction at the interface of the two media. After the linearization, in the most important particular cases of $\theta=0^{\circ}, 45^{\circ}, 90^{\circ}$, one gets for the polarization rotation $\psi$ :

$$
\begin{aligned}
\psi\left(\theta=0^{\circ}\right) & \approx \frac{\varepsilon_{x y}}{\varepsilon_{x x}} \frac{\sqrt{\varepsilon_{x x}}}{\left(\varepsilon_{x x}-1\right)} \\
\psi\left(\theta=45^{\circ}\right) & \approx \frac{\varepsilon_{x x}-\varepsilon_{y y}}{\varepsilon_{x x}} \frac{\sqrt{\varepsilon_{x x}}}{2\left(\varepsilon_{x x}-1\right)} \\
\psi\left(\theta=90^{\circ}\right) & \approx-\frac{\varepsilon_{x y}}{\varepsilon_{x x}} \frac{\sqrt{\varepsilon_{x x}}}{\left(\varepsilon_{x x}-1\right)} .
\end{aligned}
$$

Note that as soon as the aforementioned anisotropy is induced, even a subsequent isotropic excitation modifying $\varepsilon_{x x}$ and $\varepsilon_{y y}$ in the same way can lead to a rotation of the polarization plane.

The polarization plane rotation of the probe beam, in reflection or in transmission, was measured by means of a compensation scheme based on a split-diode detector and a Wollastone prism, employing a standard modulation technique with a mechanically chopped pump beam and a lock-in amplifier. We were unable to see any systematic difference between the reflection and transmission geometries, other than the signal-to-noise ratio. The latter was noticeably better for the measurements in reflection, which can be understood from the following considerations. Most of the effects discussed below are related to the top surface of the sample, be it the Co dots or the surface of the glass substrate. When measuring in reflection, the signal is collected from the thin top interface of the sample, thus the relative weight of the interface effects is increased. In contrast, for the transmission geometry, the whole bulk contributes to the registered signal, which can smear out surface effects if they are relatively small.

\section{EXPERIMENTAL RESULTS}

A typical result for the polarization rotation $\psi$ of the probe beam is presented in Fig. 2 for the polarizations of both pump and probe beams shown in the inset. In the short time range (ST), the polarization rotation demonstrates an ultrafast increase and a partial recovery. The increase and the recovery are followed by a strongly damped oscillation in the middle time range (MT). Later on another oscillatory mode emerges, exhibiting a different frequency from the previous one, which defines the long time range (LT). The time axis is broken into three distinctly outlined regions shown by colored backgrounds. We shall refer to these regions as the short (delays $\tau<$ few ps), the middle (delays of a few hundreds of ps), and the long ( $\tau>500-600 \mathrm{ps}$ ) time ranges. We shall consequently discuss all these ranges in more details in the following subsections.

\section{A. Short time range}

The probe polarization dynamics on the ultrafast timescale (below 20 ps) is presented in Fig. 3(a). The rotation $\psi$ exhibits a quick increase around zero time delay followed by a slower relaxation process. Moreover, both seem to be dependent on the polarizations of the beams. The data

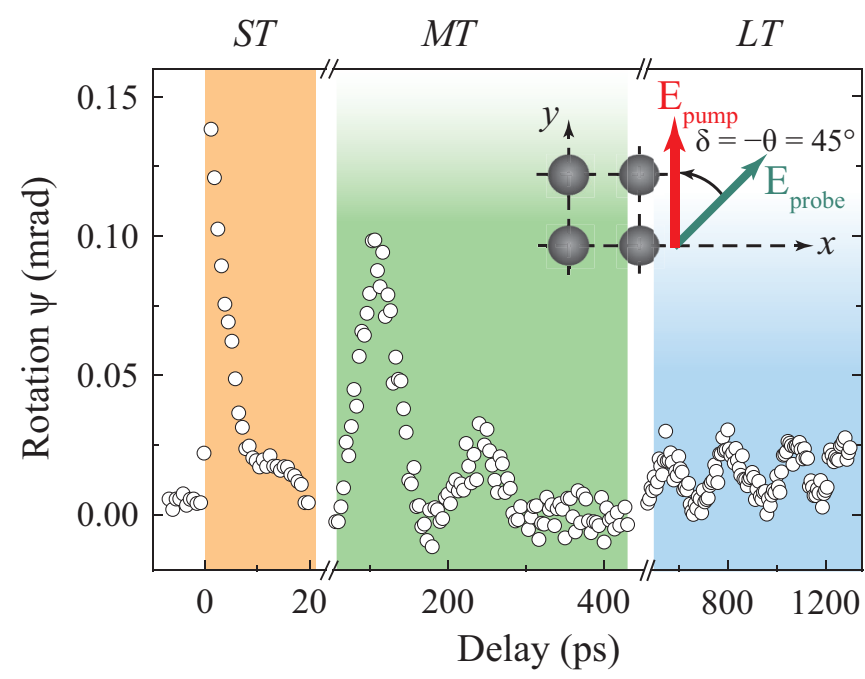

FIG. 2. (Color online) The typical dynamics of the polarization rotation of the probe beam as a function of the time delay between the pump and probe pulses. Colored background outlines three main regions in the plot, short term (ST), middle term (MT), and long term (LT) ranges. The inset shows the polarizations of both pump and probe beams with respect to the axes of the nanodots array. 

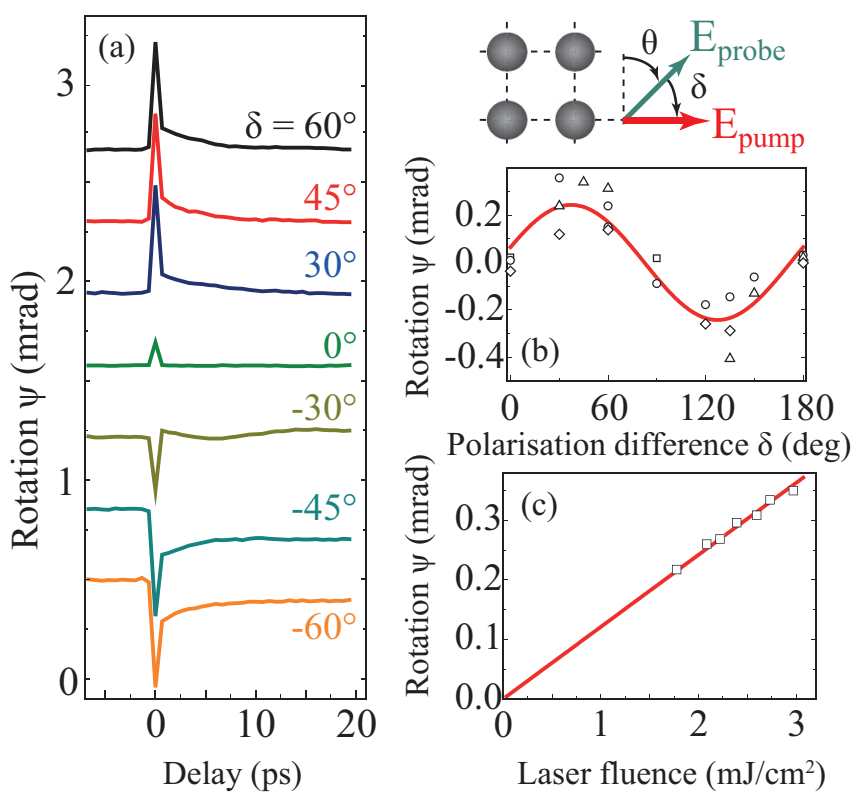

FIG. 3. (Color online) (a) Dynamics of the rotation of the polarization plane of the reflected radiation measured for different angles between the polarization planes of the probe and pump beams $\delta$ for delay times up to 20 ps. (b) The ultrafast pump-induced rotation of the polarization plane of the probe beam as a function of the angle $\delta$ between polarizations of the incident pump and probe beams. Different input probe polarizations are shown in open squares $\left(\theta=45^{\circ}\right)$, diamonds $\left(\theta=90^{\circ}\right)$, circles and triangles $\left(\theta=0^{\circ}\right)$. (c) The ultrafast pump-induced rotation of the polarization plane of the probe beam as a function of the pump laser fluence. The solid line is the linear fit to the data.

plotted here correspond to the $p$-polarized input probe beam, whereas the polarization of the pump beam was varied. Similar measurements performed for various polarizations of both beams have shown that the important parameter here is the angle $\delta$ between the polarizations of the two beams. Figure 3(b) illustrates the $180^{\circ}$ periodicity in the rotation $\psi$ as a function of $\delta$. Here the peak value of $\psi$ is plotted for a few different input probe polarizations.

It can be seen that $\psi$ vanishes for parallel or perpendicular orientations of the pump and probe polarizations $\left(\delta=0^{\circ}, 90^{\circ}\right.$, respectively). At the same time, the largest rotation on the short timescale roughly corresponds to the cases when $\delta=+45^{\circ}$ or $\delta=-45^{\circ}$. This observation can be explained in terms of the nonlinear-optical Kerr effect, where the pump radiation induces linear birefringence with the optical axis in the direction of the pump polarization. However, when considering the ultrafast Kerr nonlinearity, one should remember it is often referred to as instantaneous as it gives rise to a polarization rotation of the probe beam until the optical coherence is lost, i.e., during the excitation of the electrons. For instance, typical lifetimes for the transient nonlinear Kerr effect in metallic films and particles was found to lie in the subpicosecond range [29-32]. In our case, the effect can be separated into two contributions, one of them being ultrafast, with a characteristic time of less than a picosecond, and the other living much longer [Fig. 3(a)]. It seems natural to attribute the ultrafast part of the signal to the nonlinear-optical Kerr effect in the
Co nanodots, as free electrons there can exhibit very short response times, down to a few femtoseconds. It is generally believed that in transition metals, both interband and intraband transitions contribute to the ultrafast Kerr effect [32,33]. On this timescale the nanodots act as independent light absorbers.

For an isotropic medium, as discussed in Ref. [33], a Kerr-induced polarization rotation of a linearly polarized probe beam in the case of a linearly polarized pump beam is given by

$$
\psi=-\frac{32 \pi^{2} I_{\text {pump }}}{c|1+n|^{2}} \chi^{*} \times \sin 2 \delta,
$$

where $I_{\text {pump }}$ is the intensity of the pump beam, $c$ is the speed of light, $n$ is the complex refractive index of the material, $\chi^{*}$ is the effective third-order susceptibility, $\chi^{*}=$ $\mathbb{R} e\left(\chi_{x x y y}+\chi_{y x x y}\right) / n\left(1-n^{2}\right)$, and $\delta$ is the angle between polarizations of the pump and probe beams. Estimations of the effective susceptibility $\chi^{(3)}$ neglecting the ellipticity of the probe beam give a value of the order of $10^{-6} \mathrm{~cm}^{3} \mathrm{erg}^{-1} \mathrm{rad}^{-1}$. The measured dependence of the probe polarization rotation on the pump intensity given by Eq. (3) is shown in Fig. 3(c), where a clear linear trend can be seen.

Concerning the long-living tail of the signal, it is likely to have a different physical origin than the ultrafast contribution $[32,33]$, but instead has to do with the dielectric substrate. For instance, dislocations and defects in the substrate can be responsible for light-induced electric dipoles exhibiting a much longer lifetime than those in metals and thus can give rise to the observed effects. These dipoles sustain the initial anisotropy of the photoexcitation, thus allowing for the polarization rotation. For instance, long-living pump-induced dipole moments excited via the electrostrictive mechanism $[34,35]$ were found to govern the transient nonlinear-optical response in various dielectric media on the sub- and nanosecond timescale [36-38]. This suggestion is further confirmed by the fact that for some measurements the initial offset of the rotation $\psi$ remains for delay times of $1 \mathrm{~ns}$ and even longer, see for instance Fig. 5.

\section{B. Middle time range}

\section{Nonmagnetic effects}

Under the middle time range we shall understand relatively large time delays between 20 and 400 ps where no coherent ultrafast optical effects can be expected. In Fig. 4(a) the dynamics of the rotation $\psi$ is shown for the case when the incident probe radiation was polarized at $45^{\circ}$ with respect to the main axes of the nanodots array. Similar measurements were performed for other polarizations of both pump and probe beams. For all the cases, about two oscillation periods with slightly varying amplitudes are accompanied by a strong damping, indicating a poor quality factor of the oscillator.

It was found that although the amplitude of the oscillations varies with polarization in a way similar to that observed on the short timescale, both the phase and the frequency do not depend on the angle $\delta$ between the polarizations of the pump and probe beams. Note that the polarization of the probe was kept fixed at $\theta=45^{\circ}$. Experimental curves were fitted using a function of exponentially decaying oscillations superimposed on a steplike ultrafast increase with an exponential relaxation. 
(a)

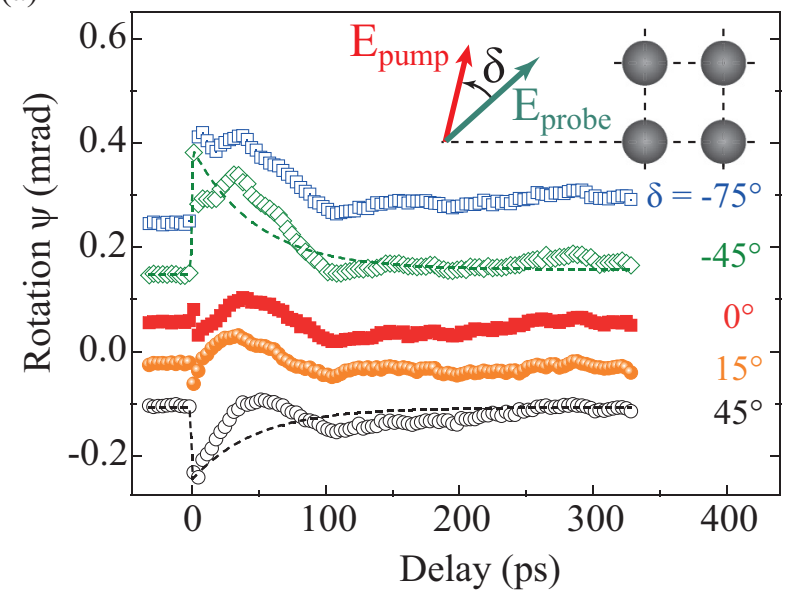

(b)

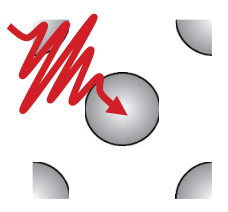

(c)

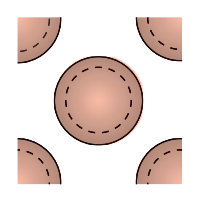

$0.1 \mathrm{ps}$

$1-300$ ps

(d)

FIG. 4. (Color online) (a) Dynamics of the rotation of the polarization plane of the reflected probe radiation for different angles between the polarization planes of the probe and pump beams for the delay times up to $300 \mathrm{ps}$. Dashed lines are the exponentially decaying contribution to the transient birefringence. (b)-(d) Excitation of the eigenmodes of Co nanodots: (b) optical pulse hits the nanodots array and is absorbed in the metal; (c) ultrafast heating leads to nonequilibrium expansion of the dots. After being driven out of equilibrium, the dots start to oscillate in-plane; (d) the excess heat sinks down to the substrate where the acoustic phonons are excited.

This exponentially decaying contribution to the transient birefringence is shown in Fig. 4 by dashed lines. The average period $T_{m}$ of the oscillations was found to be $(127 \pm 19)$ ps, corresponding to the frequency of $7.9 \mathrm{GHz}$, whereas the lifetime of the oscillations was $(139 \pm 42)$ ps.

In order to explain the observed dynamics of the optical signal, the following mechanism can be suggested [see Figs. 4(b)-4(d)]. Since the substrate is transparent for the $800 \mathrm{~nm}$ pump radiation, the energy is absorbed only in the Co nanodots [Fig. 4(b)]. This will lead to an instantaneous heating of the electrons to very high temperatures that will subsequently equilibrate with the Co lattice on the ps timescale. Because the fused quartz substrate forms a thermal bottleneck, the Co dots will be heated far above room temperature and the energy will be transferred into acoustic eigenmodes of the dots [see Fig. 4(c)]. A quantitative analysis, assuming the speed of sound in Co $c=\lambda / T_{m} \approx 4.7 \mathrm{~km} / \mathrm{s}$, yields the wavelength of the excited phonons of $0.6 \mu \mathrm{m}$, which perfectly matches the diameter of the dots [39].

This excitation corresponds to the lowest eigenmode, where the Co dots experience homogeneous in-plane expansion and contraction. In other words, a phonon with a $k$ number equal to the inverse size of the dot is excited due to the heat-driven expansion. The quantitative estimations based on the average laser fluence and specific heat capacity of Co yield the temperature increase of about $210 \mathrm{~K}$, thus not allowing the dots to overcome their Curie temperature. This heating corresponds to the lateral thermal expansion of the dots of about $1.5 \mathrm{~nm}$, or $0.25 \%$ of their size.

After the dots find themselves out of equilibrium [Fig. 4(c)], both coherent (eigenmode) and incoherent (thermal) phonons are excited, but only the former change the topology of the structure and thus the effective dielectric permittivity. As such, the optical response from the birefringent medium which stems from the slowly decaying long-living tail of the optically-induced anisotropy (see Sec. III A) turns out to be modulated with the frequency of the acoustic eigenmode of the Co dots. Note that the eigenmode itself does not induce any anisotropy and is not capable of rotating the polarization plane of the probe beam. Instead, it periodically modulates the effective $\varepsilon_{x x}$, thus inducing oscillations of the magnitude of the linear birefringence which already exists in the sample. No oscillations have been detected in the reflectivity signal within the experimental noise level. Instead, the transient reflectivity demonstrated a fast decrease of the $10^{-4}$ order of magnitude around the zero delay, followed by the subsequent slow recovery on the timescale of a few hundred picoseconds. This behavior is probably related to the electrostrictive excitation of the long-living dipoles in the substrate, as discussed in Sec. III A.

When the eigenmode decays, it means that the coherence is lost, and the energy of the eigenmode is transferred into heat and subsequently disappears into the substrate. Eventually all the excitation energy from the dots reaches the substrate [Fig. 4(d)]. This occurs at time delays of about 300 ps, where the aforementioned oscillations are followed by a flat window where no periodic dynamics of the polarization rotation is observed.

Note that on the timescale below 600 ps the dynamics of the polarization of the reflected probe beam is determined by single nanodots and not by the collective properties of their array. We shall refer to the excitation modes of a single nanodot as intradot phonons, whereas the collective modes relying on the periodicity of the nanodots will be addressed as interdot phonons. Now, at time delays of about 300 ps the excitation energy is transferred into the substrate, and intradot phonons have already decayed. In turn, collective interdot phonons with all possible $k$ are excited, propagating with the speed of sound $c$. As such, it should take at least $\Delta \tau=a / c$ for the nanodots to feel the presence of their neighbors, where $a$ is the period of the array. For the longitudinal sound wave (which is faster than the transversal) and shortest distance between the neighbors it gives the smallest $\Delta \tau$ of about $250 \mathrm{ps}$. This explains the late onset of the long range dynamics determined by the interdot phonons, which is discussed in Sec. III C.

\section{Magnetic effects}

In this subsection we shall discuss the magnetic contribution to the observed rotation of the polarization of the probe beam. Figure 5(a) shows the dependencies $\psi(\tau)$ measured for opposite directions of the longitudinal magnetic field $H= \pm 2 \mathrm{kOe}$, strong enough to saturate the magnetization. 


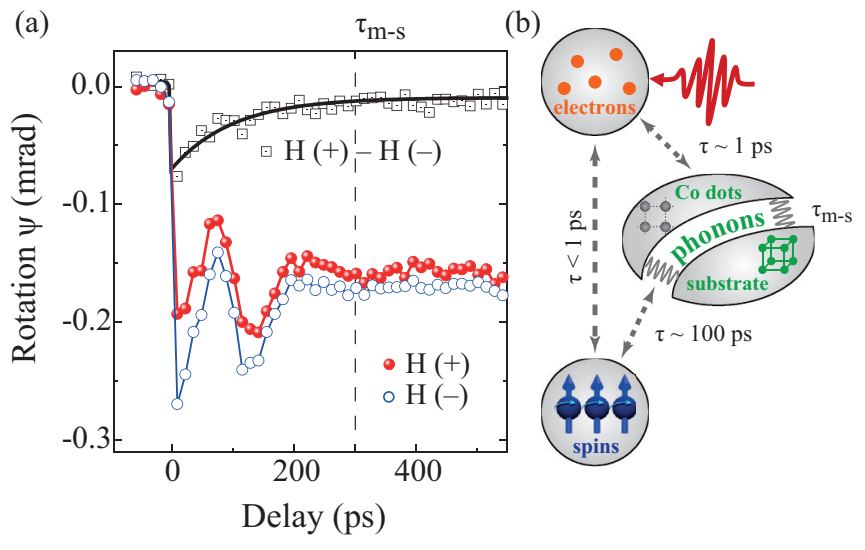

FIG. 5. (Color online) (a) Polarization rotation dynamics for two opposite directions of the longitudinal magnetic field of $\pm 2 \mathrm{kOe}$ (full red and open blue circles) together with their difference (black open squares). The solid line is an exponential fit to the experimental data. (b) Three-temperature model with characteristic times of interaction between each of the three reservoirs.

For long time delays, the dependencies $\psi(\tau)$ are very similar and demonstrate oscillations of the polarization rotation that are nearly of the same amplitude and phase, independently on the magnetic field. Thus we assume these oscillations are of a nonmagnetic nature. In contrast, magnetic field-induced modulations can be distinguished in the $\psi(\tau)$ dependencies in the short and middle delay ranges.

The purely magnetic contribution shown in the same figure with open black squares is the difference between the time dependencies measured in two magnetic fields of opposite polarities. After its initial breakdown it demonstrates an exponential relaxation with a characteristic time of 100 ps. This behavior was found to be independent on the strength of the external magnetic field which was varied in the range from $2 \mathrm{kOe}$ up to $5 \mathrm{kOe}$. The origin of the magnetic contribution to the rotation of the polarization plane is magnetic circular birefringence, which represents the magnetization dynamics of the nanodots.

Figure 5(b) illustrates the typical three-temperature model [40] conventionally used to describe the magnetization dynamics in ferromagnetic metals. The laser pulse hits the sample and within a few femtoseconds the electronic temperature rises significantly, leading to a nonequilibrium distribution of the electrons. The latter start to interact with the two other reservoirs, namely, the phonons and spins, relaxing back to their equilibrium state. These relaxation rates are material specific, which can lead to various scenarios of magnetization dynamics [41]. It was shown that in $3 d$ transition metals such as Co, the electron-spin thermalization occurs on a subpicosecond timescale leading to ultrafast demagnetization [see the quick drop in the signal right after the overlap, Fig 5(a)]. Subsequently, both electron and spin reservoirs with elevated temperatures transfer their energy to the lattice reservoir, which happens on a much longer timescale. This process is accompanied by the magnetization recovery, as the spin reservoir cools down.

Interestingly, the relaxation of the magnetization and the decay of the coherent phonon dynamics in the Co nanodots
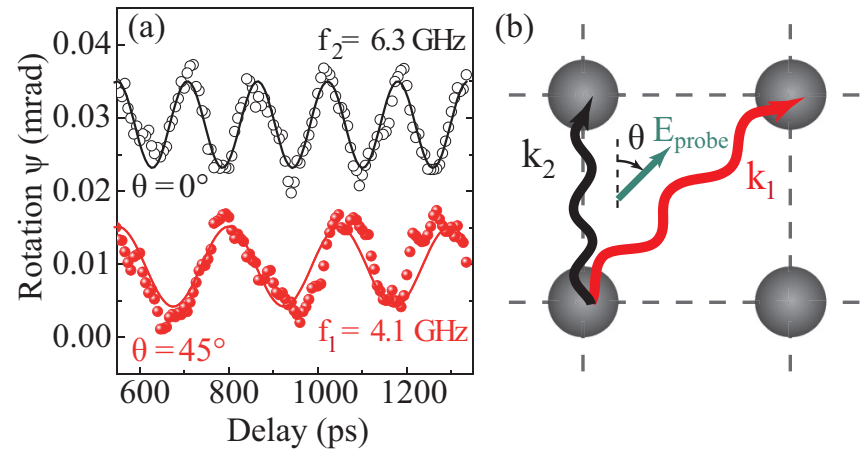

FIG. 6. (Color online) (a) Dynamics of the rotation $\psi$ of the polarization plane of the reflected radiation (long timescale) for $\theta=0^{\circ}$ and $\theta=45^{\circ}$. (b) Corresponding acoustic waves excited in the array of nanodots at $\alpha=45^{\circ}\left(k_{1}\right)$ and $\alpha=90^{\circ}\left(k_{2}\right)$.

share the same timescale. It is seen that when the oscillations of the nanodots have decayed completely [Fig. 5(a), the vertical dashed line], the magnetization is already restored to its initial value. The fit procedure demonstrated that the dynamics of both magnetization and eigenmode of a single nanodot exhibits very similar relaxation times of about 120 ps. Both processes are related to the speed of energy transfer from Co to the substrate.

\section{Long time range}

The long time range here indicates time delays longer than $400 \mathrm{ps}$, corresponding to the conditions where the dots and the substrate reach thermal equilibrium. Experimental time dependencies of the polarization rotation $\psi(\tau)$ in this range [Fig. 6(a)] demonstrate oscillations for delay times $\tau>500$ ps with frequencies of 4-6 GHz, as derived from a Fourier analysis.

Two characteristic eigenmodes with distinct frequencies $f_{1}$ and $f_{2}$ and the same phases are observed in a square lattice of the dots upon changing the input probe polarization, $(4.1 \pm$ $0.4) \mathrm{GHz}$ and $(6.3 \pm 0.5) \mathrm{GHz}$, respectively. The phase and the frequency appeared to be independent of the magnetic field direction and strength. Also, no dependence on the pump polarization was found in the experiments. The ratio of the two frequencies is close to $\sqrt{2}$. Moreover, the frequency of the oscillations demonstrates a $\pi / 2$ periodicity with respect to the input polarization, consistent with the array symmetry.

To reveal the origin of this dynamics, we note that the pump-induced oscillations are usually related to the excitation of quasiparticles [42-46], for instance, magnons or phonons. However, our experiments have shown that neither the phase of the oscillations changes upon switching the magnetic field, nor their frequency shifts when the magnetic field strength is changed (see Sec. III B). Thus we have to exclude the magnetic nature of the oscillations. Similarly, we have to exclude the excitation of optical phonons from the consideration, as these phonons usually have much higher frequencies $[47,48]$. In the same way plasmonic excitations can not be responsible for the observed oscillations, firstly due to their higher frequency $[49,50]$ and secondly, due to the extremely short lifetime of a localized plasmon which can be down to a few femtoseconds [51-54]. Also, additional 
experiments with various wavelengths of the probe beam demonstrated no significant difference in the magnitude of the effect. It turns out that the only possible explanation for the observed dynamics is the excitation of acoustic phonons which induce birefringence in the substrate. The source of this excitation is the energy of the pump beam, which is quickly (within a few femtoseconds) absorbed by the metallic nanodots and is transferred to the substrate. Generally speaking, both transversal and longitudinal acoustic waves may be generated incoherently, however, only the eigenmodes given by the array can survive long enough to contribute to the experimentally observed effects. Other excited phonons are incoherent and decay quickly.

In order to study the acoustic excitations responsible for the polarization rotation in more detail, we shall consider changes of the local optical properties of a medium induced by propagating acoustic waves. As the relevant acoustic waves propagate in the substrate, we shall consider an isotropic medium, whereas the $4 m$ symmetry of the nanodots array plays a role in the selection of possible phonon eigenmodes only.

The optical properties of a medium are described [55] by the permittivity tensor $\hat{\varepsilon}$ or by the inverse tensor $\hat{B}, \hat{\varepsilon}=\hat{B}^{-1}$. As we consider pump-induced effects to be small and our medium isotropic, after linearization one gets (see Appendix) $\Delta \varepsilon_{i j} \sim \Delta B_{i j}$, which simplifies the consideration significantly. Further, $\Delta \hat{B}$ can be obtained from the acoustic displacement $\vec{u}$ and deformation tensor $S_{i j}=\frac{1}{2}\left(\frac{\partial u_{i}}{\partial x_{j}}+\frac{\partial u_{j}}{\partial x_{i}}\right)$. After writing the tensors $\hat{S}$ and $\hat{B}$ in a short form (see Appendix), the latter can be expressed by means of the photoelastic tensor $p_{\alpha \beta}$ : $\Delta B_{\alpha}=p_{\alpha \beta} S_{\beta}$.

As such, for an acoustic wave $\vec{u}$ with a given wave vector $\vec{k}$ propagating at an angle $\alpha$ to the $x$ axis [see Fig. 6(b)] we can calculate $\Delta \varepsilon$ and thus link up the acoustic wave to the polarization rotation of the probe beam, as briefly discussed in Sec. II. Table I summarizes the results for both longitudinal and transversal waves [see also Eqs. (2), (A3), and (A4) for details].

Note that $\alpha=0^{\circ}$ (or $90^{\circ}$ ) means that the wave propagates along the main axis of the nanodots array, whereas $\alpha=45^{\circ}$ corresponds to the diagonal of the elementary array cell. As such, the spatial period of the array $a$ for $\alpha=0^{\circ}$ is shorter than that of $\alpha=45^{\circ}$, hence the $k$ vector is longer and the frequency is higher. This allows us to establish the type of acoustic waves which manifest themselves in our experiments. For the probe beam polarized along the main axes of the array of nanodots $\left(\theta=0^{\circ}, 90^{\circ}\right)$, oscillations with the higher frequency $f_{2}$ are excited [see Fig. 6(a)], which corresponds to the wave with the

TABLE I. Dielectric permittivity $\varepsilon$ components responsible for the polarization rotation of the probe beam with various input polarizations $\theta$ and corresponding contributions from the acoustic waves propagating at an angle $\alpha$ with respect to the main axes of the array. TW and LW stand for transversal waves and longitudinal waves, respectively.

\begin{tabular}{lccc}
\hline \hline$\theta$ & Rotation $\psi$ & TW & LW \\
\hline $0^{\circ}$ & $\Delta \varepsilon_{x y}$ & $\cos 2 \alpha$ & $\sin 2 \alpha$ \\
$45^{\circ}$ & $\Delta \varepsilon_{y y}-\Delta \varepsilon_{x x}$ & $\sin 2 \alpha$ & $\cos 2 \alpha$ \\
$90^{\circ}$ & $\Delta \varepsilon_{x y}$ & $\cos 2 \alpha$ & $\sin 2 \alpha$ \\
\hline \hline
\end{tabular}

larger wave vector $k_{2}$. The latter thus should be directed along one of the main axes of the array [Fig. 6(b)], which brings the $\alpha$ angle to $0^{\circ}$ (or $90^{\circ}$ ) thus leading to $\sin 2 \alpha=0$. As such, only transversal acoustic waves can contribute to this polarization rotation. Obviously, similar considerations apply to the case when the incident probe beam is polarized with $\theta=45^{\circ}$.

We can estimate the velocity with which an acoustic wave has to propagate to be able to induce the observed effects: $c=$ $f_{2} a\left(=f_{1} a \sqrt{2}\right)=8.8 \mathrm{~km} / \mathrm{s}$. This value is much higher than those known for the longitudinal $(5.98 \mathrm{~km} / \mathrm{s})$ and transversal $(3.77 \mathrm{~km} / \mathrm{s})$ waves in fused silica [56]. For a surface (Rayleigh) acoustic wave, its frequency is given by [57] $f=\xi c_{\perp} k$, where $\xi<1$ is a parameter which depends on the material properties only (for fused silica the Poisson ratio [58] $\sigma=0.164$, and thus $\xi \approx 0.9$ ) [57], and $c_{\perp}$ is the speed of the transversal sound wave. As $\xi<1$, the discrepancy between the experimental and theoretical values becomes even greater. This indicates that we are probably observing a higher harmonic of the acoustic wave, whereas the speed of sound on the silica interface appears to be modified by the Co nanodots load [20,59].

The reason why the fundamental frequency is not observed in our experiments is probably the following. When the energy from the laser pulse reaches the substrate, phonon waves with all possible wave vectors are excited. Among those which survive in the array long enough are, of course, waves with opposite values of $k$, i.e., propagating in opposite directions. Let us consider these waves propagating along the $x$ axis. According to Eq. (A3), the total phonon-induced $\Delta B$ corresponds to the standing wave, thus yielding zero contribution to the total anisotropy when averaged over a relatively large area of the laser spot. As such, the odd-order terms in the expansion $\varepsilon(B)$ can be excluded from consideration, and one should consider even ones only. The lowest one is the second-order term proportional to $B^{2}$ which matches the observed frequency within the $20 \%$ margin. The latter can be readily explained by the aforementioned load effect.

\section{CONCLUSIONS}

Summarizing, we have studied the dynamics of the polarization plane rotation induced by an intense pump laser radiation in a square lattice of magnetic nanodots. We demonstrate that the temporary behavior of the pump-induced birefringence in this structure is affected by four distinct effects. Namely, a fast Kerr nonlinear-optical effect plays the main role on the subpicosecond timescale, while at delay times of hundreds of picoseconds acoustic eigenmodes of the nanodots induce birefringence in the sample. Heat slowly diffusing down into the substrate is responsible for the late onset of the array eigenmodes, whereas transversal acoustic waves modulate the polarization of the probe beam via dynamical birefringence in the initially isotropic substrate. On top of all that, an ultrafast breakdown $(<1 \mathrm{ps})$ and relatively slow recovery $(\bumpeq 100 \mathrm{ps})$ of the magnetization in the nanodots contributes to the observed effects via Faraday or Kerr rotation.

\section{ACKNOWLEDGMENTS}

The authors are thankful to V.V. Rogov and E.V. Skorokhodov for the fabrication of the samples. This work 
was partially supported by RFBR Grant No. 13-02-01102, and financial support from the 7th Framework Program (EUFP7) Grant No. NMP-3-LA-2010-246102 (IFOX Project) is gratefully acknowledged, as well as the Program "Invited Scientist" funded by the Russian Ministry of Education and Science (Grant No. 14.B37.21.0899).

\section{APPENDIX A: FROM ACOUSTIC WAVES TO DIELECTRIC PERMITTIVITY}

Consider an acoustic wave propagating with the wave vector $\vec{k}_{a c}$ in the sample plane [see the reference frame in Fig. 6(b) in its general form:

$$
\vec{u}=\left(u_{0 x} \vec{e}_{x}+u_{0 y} \vec{e}_{y}\right) e^{i\left(k_{x} x+k_{y} y\right)}
$$

where $\vec{e}_{i}$ is a unit vector along the $i$ direction, $i=x, y, z$. This wave can be regarded as a superposition of both longitudinal and transversal waves, whereas the case of a Rayleigh surface wave will be discussed below. Assume the wave is propagating at an angle $\alpha$ to the $x$ axis. Now, in the case of a transversal acoustic wave $\left(\vec{u} \perp \vec{k}_{a c}\right)$ :

$$
\begin{gathered}
u_{0 x}=-u_{0} \sin \alpha, \quad u_{0 y}=u_{0} \cos \alpha \\
k_{x}=k \cos \alpha, \quad k_{y}=k \sin \alpha .
\end{gathered}
$$

Similarly, for a longitudinal wave $\left(\vec{u} \| \vec{k}_{a c}\right)$ :

$$
\begin{gathered}
u_{0 x}=u_{0} \cos \alpha, \quad u_{0 y}=u_{0} \sin \alpha \\
k_{x}=k \cos \alpha, \quad k_{y}=k \sin \alpha .
\end{gathered}
$$

Acoustic waves create deformation $S_{i j}=\frac{1}{2}\left(\frac{\partial u_{i}}{\partial x_{j}}+\frac{\partial u_{j}}{\partial x_{i}}\right)$, which can also be calculated. For the transversal wave one gets:

$\hat{S}_{\perp}$

$$
=i u_{0} k e^{i \vec{k} \vec{r}}\left(\begin{array}{ccc}
-\sin \alpha \cos \alpha & \frac{1}{2}\left(\cos ^{2} \alpha-\sin ^{2} \alpha\right) & 0 \\
\frac{1}{2}\left(\cos ^{2} \alpha-\sin ^{2} \alpha\right) & \sin \alpha \cos \alpha & 0 \\
0 & 0 & 0
\end{array}\right) .
$$

And for the longitudinal one:

$$
\hat{S}_{\|}=i u_{0} k e^{i \vec{k} \vec{r}}\left(\begin{array}{ccc}
\cos ^{2} \alpha & \sin \alpha \cos \alpha & 0 \\
\sin \alpha \cos \alpha & \sin ^{2} \alpha & 0 \\
0 & 0 & 0
\end{array}\right) .
$$

The deformation-induced change of the inverse dielectric permittivity $\Delta \hat{B}$ can be expressed as $\Delta B_{\alpha}=p_{\alpha \beta} S_{\beta}$, where $p_{\alpha \beta}$ is the photoelastic tensor, and we write the symmetric tensors $\hat{B}$ and $\hat{S}$ in a short way:

$$
\begin{array}{ccc}
S_{1}=S_{x x}, & S_{2}=S_{y y}, & S_{3}=S_{z z} \\
S_{4}=2 S_{x y}, & S_{5}=2 S_{x z}, & S_{6}=2 S_{y z} .
\end{array}
$$

The photoelastic tensor of an isotropic medium can be used for our purposes [55]:

$$
p=\left(\begin{array}{cccccc}
p_{11} & p_{12} & p_{12} & 0 & 0 & 0 \\
p_{12} & p_{11} & p_{12} & 0 & 0 & 0 \\
p_{12} & p_{12} & p_{11} & 0 & 0 & 0 \\
0 & 0 & 0 & p_{44} & 0 & 0 \\
0 & 0 & 0 & 0 & p_{44} & 0 \\
0 & 0 & 0 & 0 & 0 & p_{44}
\end{array}\right) .
$$

As such, from (A2) we can find $\Delta B$ :

$$
\begin{aligned}
& \Delta B_{1}=p_{11} S_{x x}+p_{12} S_{y y}+P_{12} S_{z z} \\
& \Delta B_{2}=p_{12} S_{x x}+p_{11} S_{y y}+P_{12} S_{z z} \\
& \Delta B_{3}=p_{12} S_{x x}+p_{12} S_{y y}+P_{11} S_{z z} \\
& \Delta B_{4}=2 p_{44} S_{x y} \\
& \Delta B_{5}=2 p_{44} S_{x z} \\
& \Delta B_{6}=2 p_{44} S_{y z} .
\end{aligned}
$$

Thus, for the transversal acoustic wave the induced change of the inverse dielectric permittivity $\Delta \hat{B}$ can be calculated:

$$
\Delta \hat{B}_{\perp}=\frac{1}{2} i u_{0} k e^{i \vec{k} \vec{r}}\left(\begin{array}{ccc}
\sin 2 \alpha\left(p_{12}-p_{11}\right) & 2 p_{44} \cos 2 \alpha & 0 \\
2 p_{44} \cos (2 \alpha) & -\sin 2 \alpha\left(p_{12}-p_{11}\right) & 0 \\
0 & 0 & 0
\end{array}\right)
$$

and for the longitudinal wave:

$$
\Delta \hat{B}_{\|}=\frac{1}{2} i u_{0} k e^{i \vec{k} \vec{r}}\left(\begin{array}{ccc}
p_{11} \cos ^{2} \alpha+p_{12} \sin ^{2} \alpha & p_{44} \sin 2 \alpha & 0 \\
p_{44} \sin 2 \alpha & p_{11} \sin ^{2} \alpha+p_{12} \cos ^{2} \alpha & 0 \\
0 & 0 & p_{12}
\end{array}\right) .
$$

Optical properties of a medium are described by the permittivity tensor $\varepsilon$ or by the inverse tensor $B$ [55]:

$$
\begin{aligned}
\varepsilon_{i j} B_{j k} & =\delta_{i k} \\
\hat{\varepsilon} & =\hat{B}^{-1} .
\end{aligned}
$$

Mechanical deformations result in modulations of the tensor $\mathrm{B}$, which we denote as $\Delta B$ :

$$
B=B_{0}+\Delta B \text {. }
$$

Thus the required permittivity tensor can be written as

$$
\hat{\varepsilon}=\left(\varepsilon^{-1}+\Delta B\right)^{-1},
$$


where $\varepsilon$ is the permittivity in the absence of deformation. For a small $\Delta B$, we can employ (A5) to simplify (A6) and obtain [55]:

$$
\Delta \varepsilon_{i l}=-\varepsilon_{i j} \Delta B_{j k} \varepsilon_{k l} .
$$

Here $\varepsilon_{i j}$ is the dielectric permittivity of the isotropic substrate, $\varepsilon_{i j}=\varepsilon \delta_{i j}$. As such, for the further analysis we may use:

$$
\Delta \varepsilon_{i j} \sim \Delta B_{i j} .
$$

\section{APPENDIX B: POLARIZATION ROTATION IN A BIREFRINGENT MEDIUM}

From Appendix A we see that the excitation of both transversal and longitudinal waves in a isotropic medium results in the modification of the dielectric permittivity $\hat{\varepsilon}$. The latter thus obtains the following form:

$$
\hat{\varepsilon}=\left(\begin{array}{ccc}
\varepsilon_{x x}+\Delta \varepsilon_{x x} & \Delta \varepsilon_{x y} & 0 \\
\Delta \varepsilon_{x y} & \varepsilon_{x x}+\Delta \varepsilon_{y y} & 0 \\
0 & 0 & \varepsilon_{z z}+\Delta \varepsilon_{z z}
\end{array}\right) .
$$

This means the medium becomes birefringent and thus allows for the polarization rotation of the reflected probe beam. In order to obtain the polarization rotation $\psi$ in such a medium, we should consider boundary conditions for the electric and magnetic fields of light [60].

We start from the normally incident beam polarized at an arbitrary angle $\theta$ with respect to the $O Y$ axis (see Fig. 1), so that $\vec{E}^{\text {in }}=\left\{E_{x}, E_{y}, 0\right\}$. On the boundary between air and the birefringent medium there are three waves, namely incident, transmitted, and reflected ones. The requirements of continuity of electric and magnetic fields at the interface give:

$$
\begin{gathered}
\vec{E}^{\text {in }}+\vec{E}^{r}=\vec{E}^{\operatorname{tr}}, \\
{\left[\vec{k}^{\text {in }} \times \vec{E}^{\text {in }}\right]+\left[\vec{k}^{r} \times \vec{E}^{r}\right]=\hat{n}\left[\vec{k}^{\text {tr }} \times \vec{E}^{\operatorname{tr}}\right],}
\end{gathered}
$$

where we have introduced the wave vectors of all three waves, and $\hat{n}$ is the effective matrix showing the nonorthogonality of the electric and magnetic fields of light in a birefringent medium. Its physical meaning is $\hat{n}^{2}=\hat{\varepsilon}$, and in an isotropic medium $\hat{n}$ acquires a diagonal form and reduces to the refractive index $n$.

Assuming the normal incidence of light and symmetric $\hat{n}\left(n_{x y}=n_{y x}\right)$, we get four equations (two for each $x$ and $y$ components of the fields) which can be combined in the following way:

$$
\begin{aligned}
& \vec{E}_{x}^{\text {in }}+\vec{E}_{x}^{r}=n_{x x}\left(\vec{E}_{x}^{\text {in }}-\vec{E}_{x}^{r}\right)-n_{x y}\left(\vec{E}_{y}^{\text {in }}-\vec{E}_{y}^{r}\right) \\
& \vec{E}_{y}^{\text {in }}+\vec{E}_{y}^{r}=n_{y y}\left(\vec{E}_{y}^{\text {in }}-\vec{E}_{y}^{r}\right)-n_{x y}\left(\vec{E}_{x}^{\text {in }}-\vec{E}_{x}^{r}\right) .
\end{aligned}
$$

Now we introduce the angle of polarization rotation $\psi$, as $\vec{E}^{\text {in }}=E^{\text {in }}\{\sin \theta, \cos \theta, 0\}$, and, similarly, $\vec{E}^{r}=E^{r}\{\sin (\theta+$ $\psi), \cos (\theta+\psi), 0\}$, and the reflectivity $r=E^{r} / E^{\text {in }}$, thus obtaining:

$$
\begin{aligned}
\sin \theta+r \sin (\theta+\psi)= & n_{x x}(\sin \theta-r \sin (\theta+\psi)) \\
& -n_{x y}(\cos \theta-r \cos (\theta+\psi)) \\
\cos \theta+r \cos (\theta+\psi)= & n_{y y}(\cos \theta-r \sin (\theta+\psi)) \\
& -n_{x y}(\sin \theta-r \sin (\theta+\psi)) .
\end{aligned}
$$

After expanding the trigonometrical functions and assuming small angles $\psi$ (so that $\sin \psi=\psi, \cos \psi=1$ ), one can solve the system of equations and obtain the final result:

$$
\psi=\frac{2}{Z}\left[\left(n_{y y}-n_{x x}\right) \tan \theta+n_{x y}\left(1-\tan ^{2} \theta\right)\right],
$$

where

$$
\begin{aligned}
& Z=-4 n_{x y} \tan \theta+\gamma\left(1+\tan ^{2} \theta\right), \\
& \gamma=n_{x x} n_{y y}-n_{x y}^{2}+n_{y y}-n_{x x}-1 .
\end{aligned}
$$

Since the laser-induced modifications are small, the components of the new $\hat{\varepsilon}_{i j}$ are related to the $n_{i j}$ in the following way:

$$
\begin{aligned}
\varepsilon_{x x} & =n_{x x}^{2} \\
\varepsilon_{x y} & =2 \sqrt{\varepsilon_{x x}} n_{x y} \\
\varepsilon_{y y} & =\varepsilon_{x x}+2 \sqrt{\varepsilon_{x x}}\left(n_{y y}-n_{x x}\right) .
\end{aligned}
$$

Together with (B8), this yields, after omitting small terms:

$$
\psi=\frac{\left(\varepsilon_{y y}-\varepsilon_{x x}\right) \tan \theta+\varepsilon_{x y}\left(1-\tan ^{2} \theta\right)}{\sqrt{\varepsilon_{x x}}\left(\varepsilon_{x x}-1\right)\left(1+\tan ^{2} \theta\right)},
$$

or, in a shorter form:

$$
\psi=\sqrt{\varepsilon_{x x}} \frac{\beta_{1} \tan \theta+\beta_{2}\left(1-\tan ^{2} \theta\right)}{\left(\varepsilon_{x x}-1\right)\left(1+\tan ^{2} \theta\right)},
$$

where we have introduced parameters $\beta_{1}=\frac{\varepsilon_{y y}-\varepsilon_{x x}}{\varepsilon_{x x}}, \beta_{2}=\frac{\varepsilon_{x y}}{\varepsilon_{x x}}$ responsible for the nondegeneracy of the principal axes and their in-plane rotation, respectively.

From (B9) we can consider important particular cases and obtain the rotation for the incident polarizations of $\theta=0^{\circ}$, $45^{\circ}, 90^{\circ}$ [see Eq. (2) in Sec. II]. When the incident light polarization coincides with one of the original principal axes $\left(\theta=0^{\circ}, 90^{\circ}\right)$, the rotation stems from the $\varepsilon_{x y}$ component, whereas for the case of $\theta=45^{\circ}$, the rotation is induced by the nonzero difference $\varepsilon_{y y}-\varepsilon_{x x}$.
[1] D. Chanda, K. Shigeta, T. Truong, E. Lui, A. Mihi, M. Schulmerich, P. V. Braun, R. Bhargava, and J. A. Rogers, Nat. Commun. 2, 479 (2011).

[2] Y. Nishijima, L. Rosa, and S. Juodkazis, Opt. Express 20, 11466 (2012).
[3] J. Sun, E. Timurdogan, A. Yaacobi, E. S. Hosseini, and M. R. Watts, Nature (London) 493, 195 (2013)

[4] V. M. Shalaev, Nat. Photon. 1, 41 (2007).

[5] K. Busch, G. von Freymann, S. Linden, S. F. Mingaleev, L. Tkeshelashvili, and M. Wegener, Phys. Rep. 444, 101 (2007). 
[6] J. A. Johnson, M. Grimsditch, V. Metlushko, P. Vavassori, B. Ilic, P. Neuzil, and R. Kumar, Appl. Phys. Lett. 77, 4410 (2000).

[7] A. Westphalen, K. Theis-Bröhl, H. Zabel, K. Rott, and H. Brückl, J. Magn. Magn. Mater. 302, 181 (2006).

[8] E. K. Semenova, F. Montoncello, S. Tacchi, G. Dürr, E. Sirotkin, E. Ahmad, M. Madami, G. Gubbiotti, S. Neusser, D. Grundler, F. Y. Ogrin, R. J. Hicken, V. V. Kruglyak, D. V. Berkov, N. L. Gorn, and L. Giovannini, Phys. Rev. B 87, 174432 (2013).

[9] D. Nardi, M. Travagliati, M. E. Siemens, Q. Li, M. M. Murmane, H. C. Kapteyn, G. Ferrini, F. Parmigiani, and F. Banfi, Nano Lett. 11, 4126 (2011).

[10] A. Comin, C. Giannetti, G. Samoggia, P. Vavassori, D. Grando, P. Colombi, E. Bontempi, L. E. Depero, V. Metlushko, B. Ilic, and F. Parmigiani, Phys. Rev. Lett. 97, 217201 (2006).

[11] G. Ctistis, E. Th. Papaioannou, P. Patoka, J. Gutek, P. Fumagalli, and M. Giersig, Nano Lett. 9, 1 (2009).

[12] B. Auguie and W. L. Barnes, Phys. Rev. Lett. 101, 143902 (2008).

[13] W. Cao, C. Song, T. E. Lanier, R. Singh, J. F. O’Hara, W. M. Dennis, Y. Zhao, and W. Zhang, Sci. Rep. 3, 1766 (2013).

[14] S. G. Rodrigo, S. Carretero-Palacios, F. J. Garcia-Vidal, and L. Martin-Moreno, Phys. Rev. B 83, 235425 (2011).

[15] G. A. Antonelli, H. J. Maris, S. G. Malhotra, and J. M. E. Harper, J. Appl. Phys. 91, 3261 (2002).

[16] D. H. Hurley and K. L. Telschow, Phys. Rev. B 66, 153301 (2002).

[17] P. S. Eldridge, P. G. Lagoudakis, M. Henini, and R. T. Harley, Phys. Rev. B 81, 033302 (2010).

[18] B. Borucki, H. Otto, and M. P. Heyn, J. Phys. Chem. B 108, 2076 (2004).

[19] R. Hildebrandt, M. Hegelich, H.-M. Keller, G. Marowsky, S. Hvilsted, Niels Christian Romer. Holme, and P. S. Ramanujam, Phys. Rev. Lett. 81, 5548 (1998).

[20] C. Giannetti, B. Revaz, F. Banfi, M. Montagnese, G. Ferrini, F. Cilento, S. Maccalli, P. Vavassori, G. Oliviero, E. Bontempi, L. E. Depero, V. Metlushko, and F. Parmigiani, Phys. Rev. B 76, 125413 (2007).

[21] J.-F. Robillard, A. Devos, I. Roch-Jeune, and P. A. Mante, Phys. Rev. B 78, 064302 (2008).

[22] G. F. Walsh and L. Dal Negro, Nano Lett. 13, 3111 (2013).

[23] S. Linden, F. B. P. Niesler, J. Förstner, Y. Grynko, T. Meier, and M. Wegener, Phys. Rev. Lett. 109, 015502 (2012).

[24] B. Canfield, S. Kujala, M. Kauranen, K. Jefimovs, T. Vallius, and J. Turunen, Appl. Phys. Lett. 86, 183109 (2005).

[25] L. Le Guyader, A. Kirilyuk, Th. Rasing, G. A. Wurtz, A. V. Zayats, P. F. A. Alkemade, and I. I. Smolyaninov, J. Phys. D: Appl. Phys. 41, 195102 (2008).

[26] E. Verhagen, L. Kuipers, and A. Polman, Opt. Express 17, 14586 (2009).

[27] S. E. Svyakhovskiy, A. A. Skorynin, V. A. Bushuev, S. V. Chekalin, V. O. Kompanets, A. I. Maydykovskiy, T. V. Murzina, V. B. Novikov, and B. I. Mantsyzov, J. Opt. Soc. Am. B 30, 1261 (2013).

[28] O. G. Udalov, M. V. Sapozhnikov, E. A. Karashtin, B. A. Gribkov, S. A. Gusev, E. V. Skorohodov, V. V. Rogov, A. Yu. Klimov, and A. A. Fraerman, Phys. Rev. B 86, 094416 (2012).

[29] F. Hache, D. Ricard, C. Flytzanis, and U. Kreibig, Appl. Phys. A 47, 347 (1988).

[30] Y. Hamanaka, A. Nakamura, S. Omi, N. Del Fatti, F. Vallee, and C. Flytzanis, Appl. Phys. Lett. 75, 1712 (1999).
[31] B. Koopmans, M. van Kampen, J. T. Kohlhepp, and W. J. M. de Jonge, Phys. Rev. Lett. 85, 844 (2000).

[32] V. V. Kruglyak, R. J. Hicken, M. Ali, B. J. Hickey, A. T. G. Pym, and B. K. Tanner, Phys. Rev. B 71, 233104 (2005).

[33] R. Wilks and R. J. Hicken, J. Phys.: Condens. Matter 16, 4607 (2004).

[34] Y. R. Shen, The Principles of Nonlinear Optics (John Wiley and Sons, New York, 1984).

[35] E. L. Buckland and R. W. Boyd, Opt. Lett. 21, 15, 1117 (1996).

[36] N. P. Duong, T. Satoh, and M. Fiebig, Phys. Rev. Lett. 93, 117402 (2004).

[37] O. F. J. Noordman and N. F. van Hulst, Chem. Phys. Lett. 253, 145 (1996).

[38] A. Castro, E. V. Sitzmann, D. Zhang, and K. B. Eisenthal, J. Phys. Chem. 95, 6752 (1991).

[39] Handbook of the physicochemical properties of the elements, edited by G. V. Samsonov (IFI-Plenum, New York, 1968).

[40] S. I. Anisimov, B. L. Kapeliovich, and T. L. Perelman, Zh. Exp. Teor. Fiz. 66, 776 (1974) [Sov. Phys. JETP 39, 375 (1974)].

[41] B. Koopmans, G. Malinowski, F. Dalla Longa, D. Steiauf, M. Fähnle, T. Roth, M. Cinchetti, and M. Aeschlimann, Nat. Mater. 9, 259 (2010).

[42] R. D. Averitt, Nat. Phys. 4, 14 (2008).

[43] A. Melnikov, I. Radu, U. Bovensiepen, O. Krupin, K. Starke, E. Matthias, and M. Wolf, Phys. Rev. Lett. 91, 227403 (2003).

[44] R. A. Kaindl, M. Woerner, T. Elsaesser, D. C. Smith, J. F. Ryan, G. A. Farnan, M. P. McCurry, and D. G. Walmsley, Science 287, 470 (2000).

[45] J. Demsar, K. Biljakovic, and D. Mihailovic, Phys. Rev. Lett. 83, 800 (1999).

[46] J. Graf, C. Jozwiak, C. L. Smallwood, H. Eisaki, R. A. Kaindl, D.-H. Lee, and A. Lanzara, Nat. Phys. 7, 805 (2011).

[47] J. M. Ziman, Electrons and Phonons. The Theory of Transport Phenomena in Solids (Clarendon Press, Oxford, 1960).

[48] A. Laubereau and W. Kaiser, Rev. Mod. Phys. 50, 607 (1978).

[49] H. Raether, Surface Plasmons (Springer-Verlag, Berlin Heidelberg, 1988).

[50] A. V. Zayats, I. I. Smolyaninov, and A. A. Maradudin, Phys. Rep. 408, 131 (2005).

[51] M. Simon, F. Träger, A. Assion, B. Lang, S. Voll, and G. Gerber, Chem. Phys. Lett. 296, 579 (1998).

[52] B. Lamprecht, G. Schider, R. T. Lechner, H. Ditlbacher, J. R. Krenn, A. Leitner, and F. R. Aussenegg, Phys. Rev. Lett. 84, 4721 (2000).

[53] J. Lehmann, M. Merschdorf, W. Pfeiffer, A. Thon, S. Voll, and G. Gerber, Phys. Rev. Lett. 85, 2921 (2000).

[54] A. Anderson, K. S. Derycks, X. G. Xu, G. Steinmeyer, and M. B. Raschke, Nano Lett. 10, 2519 (2010).

[55] E. Dieulesaint and D. Royer, Elastic Waves in Solid Bodies. Application for signal processing (Nauka, Moscow, 1982).

[56] Ch. Matteï, X. Jia, and G. Quentin, J. Acoust. Soc. Am. 102, 1532 (1997).

[57] L. D. Landau and E. M. Lifschitz, Theory of Elasticity (Pergamon Press, Oxford, 1970).

[58] W. J. Tropf, M. E. Thomas, T. J. Harris, Properties of Crystals and Glasses, in M. Bass, Handbook of Optics, Vol. 2, 2nd ed. (McGraw-Hill, Inc., New York, 1995).

[59] C. Thoma and Y. Hahn, J. Appl. Phys 79, 8230 (1996).

[60] J. D. Jackson, Classical Electrodynamics, 3rd ed. (John Wiley \& Sons, Inc., New York, 1999). 